Bulletin of the Section of Logic

Volume 49/3 (2020), pp. 269-290

http://dx.doi.org/10.18778/0138-0680.2020.17

Simin Saidi Goraghani

Rajab Ali Borzooei

\title{
MODULE STRUCTURE ON EFFECT ALGEBRAS
}

\begin{abstract}
In this paper, by considering the notions of effect algebra and product effect algebra, we define the concept of effect module. Then we investigate some properties of effect modules, and we present some examples on them. Finally, we introduce some topologies on effect modules.
\end{abstract}

Keywords: Effect algebra, product effect algebra, effect module, topology.

2010 Mathematical Subject Classification: 06G12, 08A55, 16D80, 54H12.

\section{Introduction}

In 1994, Foulis and Bennett [16] introduced the concept of effect algebras with a partially defined addition "+" in order to axiomatize some quantum measurements. They are additive counterparts to $D$-posets introduced by Kôpka and Chovanec (1994), where the subtraction of comparable elements is a primary notion. They met interest of mathematicians physicits while they give a common base for algebraic as well as fuzzy set properties of the system $\varepsilon(H)$ of all effects of a Hilbert space $H$, i.e., of all Hermitian operators $A$ on $H$ such that $O \leq A \leq I$, where $O$ and $I$ are the null and the identity operators on $H$. In many cases, effect algebras are intervals in unital po-groups, e.g., $\varepsilon(H)$ is the interval in the po-group $\beta(H)$ of all Hermitian operators on $H$; this group is of great importance for physics.

Effect algebras generalize many examples of quantum structures, like Boolean algebras, orthomodular lattices or posets, orthoalgebras, $M V$ algebras and etc. We recall that $M V$-algebras are algebraic counterparts

Presented by: Janusz Ciuciura

Received: September 23, 2019

Published online: August 15, 2020

(C) Copyright for this edition by Uniwersytet Łódzki, Łódź 2020 
of the many-valued reasoning, and they appeared in mathematics under many different names, situations and motivations. Even in the theory of effect algebras, they were defined in an equivalent way as phi-symmetric effect algebras [1]. The monograph $[2,11]$ can serve as a basic source of information about effect algebras. Product effect algebras, were introduced by Anatolij Dvurecenskij [12]. He proved every product effect algebra with the Riesz decomposition property $(R D P)$ is an interval in an Abelian unital interpolation po-ring, and he showed that the category of product effect algebras with the $R D P$ is categorically equivalent with the category of unital Abelian interpolation po-rings. Recently, some researchers worked on modular structures (see, for instance, [3, 4, 9, 10, 17]). Effect modules have been introduced in theoretical physics in the mid-1990 for quantum probability. These structures are effect algebras with a scalar multiplication, with scalars from $[0,1]$, i.e., an effect module $M$ is an effect algebra with an action $[0,1] \times M \longrightarrow M$ that it is an special case. In this paper, we try to present more complete definition than the previous definition. We define effect modules on product effect algebras as an extension of effect algebras.

In the study of effect algebras (or more general, quantum structures) as carriers of states and probability measures, an important tool is the study of topologies on them. In fact, algebra and topology, the two fundamental domains of mathematics, play complementary roles. Topology studies continuity and convergence, and it provides a general framework to study the concept of a limit. Algebra studies all kinds of operations and provides a basis for algorithms and calculations. Because of this difference in nature, algebra and topology to have a strong tendency to develop independently, not in direct contact with each other. However, in applications, in higher level domains of mathematics, such as functional analysis, dynamical systems, representation theory and others, topology and algebra come in contact most naturally. Recently, many mathematicians have studied properties of some algebraic structures endowed with a topology (see, for instance, $[5,6,7,15,18])$. We have studied and try to introduce some topologies on effect modules. In fact, we wish to open new fields to anyone that is interested to studying and development of effect algebras and effect modules. 


\section{Preliminaries}

In this section, we review some definitions and related lemmas and theorems that we use in the next sections.

Definition 2.1 ([16]). An effect algebra is a partial algebra $E=(E ;+, 0,1)$ with a partially defined operation " +" and two constant elements 0 and 1 such that, for all $a, b, c \in E$,

(E1) Commutative Law: if $a+b$ is defined in $E$, then $b+a$ is defined in $E$, and in such the case $a+b=b+a$;

(E2) Associative Law: if $a+(b+c)$ and $b+c$ are defined in $E$, then $a+b$ and $(a+b)+c$ are defined in $E$, and in such the case $a+(b+c)=$ $(a+b)+c$;

(E3) Orthocomplementation Law: for any $a \in E$, there exists a unique element $a^{\prime} \in E$ such that $a+a^{\prime}=1$;

(E4) Zero-Unit Law: if $a+1$ is defined in $E$, then $a=0$.

The algebraic structure $(E ;+, 0)$ is called an extended effect algebra if

$(G E 1) E$ is a partial commutative monoid;

$(G E 2) x+z=x+y$ implies $z=y$;

(GE3) $x+y=0$ implies $x=y=0$, for every $x, y, z \in E$ (see [11]).

Let $E$ be an effect algebra. If we define $a \leq b$ if and only if there exists an element $c \in E$ such that $a+c=b$, then $\leq$ is a partial ordering, and we write $c:=b-a$. A nonempty subset $I$ of $E$ is said to be an ideal of $E$ if the following conditions are satisfied: $\left(I d_{1}\right)$ If $x \in I$ and $y \leq x$, then $y \in I$, $\left(I d_{2}\right)$ if $x-y \in I$ and $y \in I$, then $x \in I$, for any $x, y \in E$. Recall that a set $Q \subseteq E$ is called a sub-effect algebra of the effect algebra $E$, if $1 \in Q$ and if out of elements $a, b, c \in E$ with $a+b=c$ two are in $Q$, then $a, b, c \in Q$. Let $F$ be another effect algebra. A mapping $h: E \longrightarrow F$ is said to be a homomorphism of effect algebras (or $E$-homomorphism) if $h(1)=1$ and $h(a+b)=h(a)+h(b)$, for any $a, b \in E$ whenever $a+b$ is defined in $E$.

We say $E$ fulfills the strong Riesz Decomposition Property, (RDP2) for short, if $a_{1}, a_{2}, b_{1}, b_{2} \in P$ such that $a_{1}+a_{2}=b_{1}+b_{2}$, then there are $d_{1}, d_{2}, d_{3}, d_{4} \in P$ such that $(i) d_{1}+d_{2}=a_{1}, d_{3}+d_{4}=a_{2}, d_{1}+d_{3}=b_{1}$, $d_{2}+d_{4}=b_{2}$, and $(i i) d_{2} \wedge d_{3}=0$ (see [13]). 
Definition $2.2([12])$. A product on effect algebra $E=(E ;+, 0,1)$ is any total binary operation "." on $E$ such that for all $a, b, c \in E$, the following holds:

If $a+b$ is defined in $E$, then $a . c+b . c$ and $c . a+c . b$ exist in $E$ and

$$
(a+b) . c=a . c+b . c, \quad c .(a+b)=c . a+c . b .
$$

Now, an effect algebra $E$ with a product "." is called a product effect algebra.

The product "." on $E$ is associative if $(a . b) . c=a .(b . c)$, for every $a, b, c \in E$.

A mapping $h: E \longrightarrow F$ is said to be a homomorphism of product effect algebras (or $P$-homomorphism) if $h$ ia an $E$-homomorphism and $h(a . b)=$ $h(a) . h(b)$, for every $a, b \in P$.

Proposition 2.3 ([16]). The following properties hold for any effect algebra $E$ :

(i) $a^{\prime \prime}=a$,

(ii) $1^{\prime}=0$ and $0^{\prime}=1$,

(iii) $0 \leq a \leq 1$,

(iv) $a+0=a$,

(v) $a+b=0 \Rightarrow a=b=0$,

(vi) $a \leq a+b$,

(vii) $a \leq b \Rightarrow b^{\prime} \leq a^{\prime}$,

(viii) $b-a=\left(a+b^{\prime}\right)^{\prime}$,

(ix) $a+b^{\prime}=(b-a)^{\prime}$,

(x) $a=a-0$,

(xi) $a-a=0$,

(xii) $a^{\prime}=1-a$ and $a=1-a^{\prime}$, for every $a, b \in E$.

Definition 2.4 ([8]). An $M V$-algebra is a structure $M=\left(M, \oplus,{ }^{\prime}, 0\right)$ of type $(2,1,0)$ that satisfies the following axioms:

$(M V 1)(M, \oplus, 0)$ is an Abelian monoid, 
$(M V 2)\left(a^{\prime}\right)^{\prime}=a$,

$(M V 3) 0^{\prime} \oplus a=0^{\prime}$,

$(M V 4)\left(a^{\prime} \oplus b\right)^{\prime} \oplus b=\left(b^{\prime} \oplus a\right)^{\prime} \oplus a$.

An $l$-group is an algebra $(G,+,-, 0, \vee, \wedge)$, where the following properties hold:

(a) $(G,+,-, 0)$ is a group,

(b) $(G, \vee, \wedge)$ is a lattice,

(c) $x \leq y$ implies that $b+x+a \leq b+y+a$, for any $x, y, a, b \in G$.

A strong unit $u>0$ is a positive element with property that for any $g \in G$ there exits $n \in \omega$ such that $g \leq n u$. The Abelian $l$-groups with a strong unit will be simply called $l u$-groups.

The category whose objects are $M V$-algebras and whose homomorphisms are $M V$-homomorphisms is denoted by $M V$. The category whose objects are pairs $(G, u)$, where $G$ is an Abelian $l$-group and $u$ is a strong unit of $G$ and whose homomorphisms are l-group homomorphisms is denoted by $U g$. The functor that establishes the categorical equivalence between $M V$ and $U g$ is

$$
\Gamma: U g \longrightarrow M V,
$$

where $\Gamma(G, u)=[0, u]_{G}$, for every $l u$-group $(G, u)$ and $\Gamma(h)=\left.h\right|_{[0, u]}$, for every $l u$-group homomorphism $h$. The above results allows us to consider an $M V$-algebra, when necessary, as an interval in the positive cone of an l-group.

Definition 2.5 ([9]). A product $M V$-algebra (or $P M V$-algebra, for short) is a structure $A=\left(A, \oplus, .,^{\prime}, 0\right)$, where $\left(A, \oplus,,^{\prime}, 0\right)$ is an $M V$-algebra and "." is a binary associative operation on $A$ such that the following property is satisfied: if $x+y$ is defined, then $x . z+y \cdot z$ and $z \cdot x+z \cdot y$ are defined and $(x+y) \cdot z=x \cdot z+y . z, z \cdot(x+y)=z \cdot x+z \cdot y$, for every $x, y, z \in A$, where " + " is the partial addition on $A$.

Let $A=\left(A, \oplus, .{ }^{\prime}, 0\right)$ be a $P M V$-algebra, $M=\left(M, \oplus,^{\prime}, 0\right)$ be an $M V$ algebra and the operation $\Phi: A \times M \longrightarrow M$ be defined by $\Phi(a, x)=: a x$, which satisfies the following axioms:

$(A M 1)$ If $x+y$ is defined in $M$, then $a x+a y$ is defined in $M$ and $a(x+y)=$ $a x+a y$, 
(AM2) If $a+b$ is defined in $A$, then $a x+b x$ is defined in $M$ and $(a+b) x=$ $a x+b x$,

(AM3) $(a . b) x=a(b x)$, for every $a, b \in A$ and $x, y \in M$.

Then $M$ is called a (left) $M V$-module over $A$ or briefly an $A$-module.

We say that $M$ is a unitary $M V$-module if $A$ has a unity for the product and

(AM4) $1_{A} x=x$, for every $x \in M$.

\section{Effect modules}

In this section, we present the definition of an effect module in effect algebras and state some results on them.

Definition 3.1. Let $P=(P ;+, ., 0,1)$ be a product effect algebra and $E=(E ;+, 0,1)$ be an effect algebra. Then we say that $E$ is an effect module over $P$ or $P$-module if there is an external operation $\varphi: P \times E \longrightarrow E$, with $\varphi(a, x)=: a x$ such that for any $x, y \in E$ and $a, b \in P$, the following properties hold:

(PE1) If $a+b$ is defined, then $a x+b x$ is defined and $(a+b) x=a x+b x$.

$(P E 2)$ If $x+y$ is defined, then $a x+a y$ is defined and $a(x+y)=a x+a y$.

(PE3) $(a . b) x=a(b x)$.

Moreover, if $\varphi(1, x)=1 x=x$, for every $x \in E$, then $E$ is called a unitary $P$-module.

Example 3.2.

(i) Let $P$ be a product effect algebra and $E$ be an effect algebra. If we define $\varphi(a, x)=0$, for any $a \in P$ and $x \in E$, then $E$ becomes a $P$-module.

(ii) Consider the real unit interval $[0,1]$. Let $x \oplus y=\min \{x+y, 1\}$, for all $x, y \in[0,1]$. Then $([0,1], \oplus, 0,1)$ is an effect algebra, where "+" and " - " are the ordinary operations in $\mathbb{R}$. Moreover, consider $a b=a . b$, for every $a, b \in[0,1]$, where "." is the ordinary operation in $\mathbb{R}$. Then $[0,1]$ is a $[0,1]$-module. 
(iii) Let $E=\{0,1,2,3\}$ and the operation " + " is defined on $P$ as follows:

\begin{tabular}{c|cccc}
+ & 0 & 1 & 2 & 3 \\
\hline 0 & 0 & 1 & 2 & 3 \\
1 & 1 & - & 3 & - \\
2 & 2 & 3 & - & - \\
3 & 3 & - & - & -
\end{tabular}

Then $(E ;+, 0,3)$ is an effect algebra. If we define operation "." by

\begin{tabular}{l|llll}
. & 0 & 1 & 2 & 3 \\
\hline 0 & 0 & 0 & 0 & 0 \\
1 & 0 & 1 & 0 & 1 \\
2 & 0 & 0 & 2 & 2 \\
3 & 0 & 1 & 2 & 3
\end{tabular}

then $P=(E ;+, ., 0,3)$ is a product effect algebra. Consider $\phi(a, x)=$ a.x, for every $a, x \in E$. Then $E$ is a $P$-module.

(iv) Let $L=\{0, x, 1\}, P=\{0,1\}$ and operations + and $+^{\prime}$ is defined on $L$ and $P$, respectively, as follows:

\begin{tabular}{c|ccc}
+ & 0 & $x$ & 1 \\
\hline 0 & 0 & $x$ & 1 \\
$x$ & $x$ & 1 & - \\
1 & 1 & - & -
\end{tabular}

\begin{tabular}{c|cc}
$+^{\prime}$ & 0 & 1 \\
\hline 0 & 0 & 1 \\
1 & 1 & -
\end{tabular}

Then $(L ;+, 0,1)$ is an effect algebra and $\left(P ;+^{\prime}, ., 0,1\right)$ is a product effect algebra, where "." is the ordinary operation in $\mathbb{R}$. Consider $E=L \times L$. Then $(E ; \oplus,(0,0),(1,1))$ is an effect algebra, where $\left(e_{1}, e_{2}\right) \oplus\left(b_{1}, b_{2}\right)=\left(e_{1}+b_{1}, e_{2}+b_{2}\right)$, for every $e_{1}, e_{2}, b_{1}, b_{2} \in L$. Now, for any $a \in P$ and $\left(e_{1}, e_{2}\right) \in E$, we consider $\varphi\left(a,\left(e_{1}, e_{2}\right)\right)=\left(a e_{1}, a e_{2}\right)$, where for every $e \in L$,

$$
a e= \begin{cases}0 & a=0 \\ e & a=1\end{cases}
$$

Then $E$ is a $P$-module.

Lemma 3.3. Every associative product effect algebra $(P,+, ., 0,1)$ is a $P$ module. 
Proof: If we define $\phi(a, b)=a . b$, for every $a, b \in P$, then it is easy to see that $P$ is a $P$-module.

Proposition 3.4. Let $E$ be an effect algebra such that for every $a, b \in E$, $a^{\prime}+\left(b+a^{\prime}\right)^{\prime}=b^{\prime}+\left(a+b^{\prime}\right)^{\prime}$. Then $E$ can become an $E$-module.

Proof: If we define $a . b=(a * b)^{\prime}$, where $a * b=a^{\prime}+\left(b+a^{\prime}\right)^{\prime}$, then in a straightforward way, $E$ is an associative product effect algebra and so by Lemma $3.3, E$ is an $E$-module.

Note. Let $E=(E ;+, 0,1)$ be an effect algebra. Then for any $a, b \in E$, $a \leq b^{\prime}$ if and only if $a+b$ is defined in $E$.

Lemma 3.5. Let $E=(E ;+, 0,1)$ be an effect algebra. Then the set of

$$
\operatorname{End}(E)=\{f: E \rightarrow E: f \text { is an E-homomorphism }\}
$$

is a product effect algebra.

Proof: We consider $f+g: E \rightarrow E$, by $(f+g)(x)=f(x)+g(x)$, where $f(x)+g(x)$ is defined in $E$ and $(f+g)(x)=0$, where $f(x)+g(x)$ is not defined in $E$. Also, we consider $f \circ g: E \rightarrow E$, by $(f \circ g)(x)=f(g(x))$. Let $x+y$ be defined in $E$. Since $f, g$ are $E$-homomorphism, $f(x)+f(y)$ and $g(x)+g(y)$ are defined and so it is easy to see that $f+g$ and $f \circ g$ are $E$-homomorphism. Thus, it is routine to see that $(\operatorname{End}(E),+, \circ, I, O)$ is an effect algebra, where $I: E \rightarrow E$ and $O: E \rightarrow E$ are identity $E$ homomorphism and zero $E$-homomorphism, respectively.

TheOREM 3.6. Let $E=(E ;+, 0,1)$ be an effect algebra and $P$ be a product effect algebra. Then $E$ is a unitary $P$-module if and only if there exists a $P$-homomorphism $\varphi: P \rightarrow \operatorname{End}(E)$.

Proof: Let $E$ be a unitary $P$-module with module multiplication $\psi$ : $P \times E \rightarrow E$, by $\psi(a, x)=a x$, for every $a \in P$ and $x \in E$. By Lemma $3.5, \operatorname{End}(E)$ is a product effect algebra. We consider the function $\varphi: P \rightarrow$ $\operatorname{End}(E)$, by $a \rightarrow \varphi(a)$, where $\varphi(a): E \rightarrow E$ is defined by $\varphi(a)(x)=a x$, for every $a \in P$ and $x \in E$. We show that $\varphi$ is a homomorphism of product effect algebras. Let $a+b$ be defined in $P$, for any $a, b \in P$. Then we have

$$
\varphi(a+b)(x)=(a+b) x=a x+b x=\varphi(a)(x)+\varphi(b)(x)=(\varphi(a)+\varphi(b))(x)
$$

for every $x \in E$. It results that $\varphi(a+b)=\varphi(a)+\varphi(b)$. Now, for every $a, b \in P$, since 
$\varphi(a . b)(x)=(a . b) x=a(b x)=a(\varphi(b)(x))=\varphi(a)(\varphi(b)(x))=(\varphi(a) \circ \varphi(b))(x)$

for every $x \in E$, we have $\varphi(a . b)=\varphi(a) \circ \varphi(b)$. Also, $\varphi(1)(x)=1 x=x$, for every $x \in E$ and so $\varphi(1)=I$.

Conversely, let there is a $P$-homomorphism $\varphi: P \rightarrow E n d(E)$. We define $\psi: P \times E \rightarrow E$, by $\psi(a, x)=a x=\varphi(a)(x)$, for every $a \in P$ and $x \in E$. It is easy to see that $\psi$ is well defined.

(PE1) Let $a+b$ be defined in $P$. Then $a \leq b^{\prime}$ and so $a x \leq b^{\prime} x$. We must show that $a x+b x$ is defined in $E$. The first, we show that $b^{\prime} x \leq(b x)^{\prime}$. Since $x \leq x$, hence $x^{\prime}+x$ is defined and so

$$
b\left(x+x^{\prime}\right)=\psi(b)\left(x^{\prime}+x\right)=\psi(b)\left(x^{\prime}\right)+\psi(b)(x)=b x^{\prime}+b x
$$

Then

$$
b x^{\prime}+b x=b\left(x+x^{\prime}\right)=b 1 \leq 1=(b x)^{\prime}+b x
$$

and so $b^{\prime} x \leq(b x)^{\prime}$. Thus, $a x \leq b^{\prime} x \leq(b x)^{\prime}$ and so $a x+b x$ is defined. Hence, it is easy to see that $(a+b) x=a x+b x$.

$(P E 2)$ Let $x+y$ be defined in $E$. Similar to $(P E 1)$, we can show that $a(x+y)=a x+a y$.

(PE3) Let $a, b \in P$ and $x \in E$. Then

$$
\begin{aligned}
(a . b) x & =\psi(a . b)(x)=(\psi(a) \circ \psi(b))(x)=\psi(a)(\psi(b)(x))=\psi(a)(b x) \\
& =a(b x)
\end{aligned}
$$

Moreover, $1 x=\psi(1)(x)=x$, for every $x \in E$. Therefore, $E$ is a unitary $P$-module.

\section{TheOREM 3.7.}

(i) Every $M V$-module can be transformed into an effect module.

(ii) Every effect module satisfying (RDP2) can be transformed into an $M V$-module.

\section{PROOF:}

(i) Let $M$ be an $A$-module, where $A$ is a $P M V$-algebra. We can consider $M=\Gamma(G, u)$, where $G$ is an Abelian $l$-group and $u$ is a strong unit of $G$. Define "+" to be a partial operation on $M$ that is defined 
for elements $a, b \in M$ if and only if $a \leq b^{\prime}$, and in that case let $a+b:=a \oplus b$. Then $(M,+, 0,1)$ is an effect algebra. Similarly, $A$ can be transformed into a product effect algebra. Now, by $M V$-module multiplication, $M$ will be an effect module.

(ii) Let $E$ be a $P$-module satisfying $(R D P 2)$. By ([14], Theorem 8.8), $E$ and $P$ are $M V$-algebras. If we consider $a \bullet b=a . b$, for every $a, b \in P$, where "." is the product operation in $P$, then $P$ is a $P M V$-algebra. Now, by effect module multiplication, $E$ can be transformed into an $M V$-module.

In the rest of this paper, we let $P$ be a product effect algebra and $E$ be an effect algebra, unless otherwise specified. Also, if we are not sure that $a+b$ is defined in effect algebra $E$, then we denote $a \oplus b$ instead of $a+b$, for any $a, b \in E$.

\section{Some topologies on effect modules}

In this section, we introduce five topologies on effect modules.

Definition 4.1. Let $E$ be a $P$-module. Then $\emptyset \neq I \subseteq E$ is called a submodule of $E$ if it satisfies the following conditions, for every $a \in P$ and $x, y \in E:$

$\left(I_{1}\right)$ If $x, y \in I$ and $x+y$ is defined in $E$, then $x+y \in I$.

$\left(I_{2}\right)$ If $x \leq y$ and $y \in I$, then $x \in I$.

$\left(I_{3}\right)$ If $x \in I$, then $a x \in I$.

$I \subseteq E$ is called a $W$-submodule (weak submodule) of $E$ if it satisfying $\left(I_{3}\right)$. $I \subseteq E$ is called an $E$-ideal of $E$ if it satisfying $\left(I_{1}\right)$ and $\left(I_{2}\right)$.

We denote by $S b_{P}(E)$ and $W S b_{P}(E)$, respectively, the set of all submodules of $P$-module $E$ and the set of all $W$-submodules of $P$-module $E$. Example 4.2.

(i) For every effect module $E,\{0\}$ and $E$ are trivial submodules of $E$.

(ii) In Example $3.2($ iii $), I=\{0,1\}$ and $J=\{0,2\}$ are submodules of $E$.

(iii) Every submodule of $E$ is a $W$-submodule ( an $E$-ideal) of $E$. 
For every subset $I$ of $E$, we denote

$$
U_{I}=\{\varphi(a, x): a \in(I: E) \text { or } x \in I\},
$$

where $(I: E)=\{x \in P: x E \subseteq I\}$.

Proposition 4.3. Let $E$ be a $P$-module. Then

(i) If $I \subseteq J$, then $(I: E) \subseteq(J: E)$, where $I, J$ be subsets of $E$.

(ii) $\bigcap_{i \in I}\left(J_{i}: E\right)=\left(\bigcap_{i \in I} J_{i}: E\right)$, where $J_{i}$ is a subset of $E$, for every $i \in$ $I$.

(iii) If $I$ is a submodule of $E$, then $(I: E)$ is an ideal of $P$.

If $I$ is a $W$-submodule of $P$ as $P$-module, where $P$ is an associative product effect algebra, then

(iv) $I \subseteq(I: P)$.

(v) $(v)(I: P) P \subseteq I$.

Proof: The proof is easy.

Theorem 4.4. Let $E$ be a unitary $P$-module and $a . a=a$, for every $a \in P$. Then $\Gamma=\left\{U_{I}: I \in W S b_{P}(E)\right\}$ is a topology on $E$.

Proof: Let $E$ be a $P$-module, $a . a=a$, for every $a \in P$ and $I, J \in$ $W S b_{P}(E)$. First we prove that:

(i) $U_{\emptyset}=\emptyset$ and $U_{E}=E$.

(ii) $U_{I} \cap U_{J}=U_{I \cap J}$.

(iii) $U_{I} \cup U_{J}=U_{I \cup J}$.

The proof of (i) is clear. For the proof of (ii), since $I \cap J \subseteq I$ and $I \cap J \subseteq J$, it is easy to see that $U_{I \cap J} \subseteq U_{I} \cap U_{J}$. Let $\phi(a, x) \in U_{I} \cap U_{J}$. Then $a x \in U_{I}$ and $a x \in U_{J}$. It results that $a \in(I: E)$ or $x \in I$ and $a \in(J: E)$ or $x \in J$. There are four possible cases:

(1) If $a \in(I: E)$ and $a \in(J: E)$, then it is easy to see that $a \in(I \cap J: E)$ and so $\phi(a, x)=a x \in U_{I \cap J}$.

(2) If $x \in I$ and $x \in J$, then $x \in I \cap J$ and so $a x \in U_{I \cap J}$. 
(3) Let $a \in(I: E)$ and $x \in J$. Then $a E \subseteq I$ and so $a x \in I$. Since $J$ is a $W$-submodule of $E$, $a x \in J$, too. Hence $\phi(a, x)=a x \in I \cap J$ and so $a(a x)=\phi(a, a x) \in U_{I \cap J}$. It results that by $(P E 3)$,

$$
\phi(a, x)=a x=(a . a) x=a(a x) \in U_{I \cap J}
$$

(4) If $x \in I$ and $a \in(J: E)$, then similar to (3), we have $a x \in U_{I \cap J}$. Therefore, $U_{I} \cap U_{J} \subseteq U_{I \cap J}$.

(iii) Since $I \subseteq I \cup J$ and $J \subseteq I \cup J$, it is easy to show that $U_{I} \cup U_{J} \subseteq U_{I \cup J}$. Let $\phi(a, x) \in U_{I \cup J}$. Then $a \in(I \cup J: E)$ or $x \in I \cup J$. If $a \in(I \cup J: E)$, then $a E \subseteq I \cup J$ and so $a x \in I \cup J$. Thus, $a x \in I$ or $a x \in J$ and so $a x=a(a x) \in U_{I}$ or $a x=a(a x) \in U_{J}$. It follows that $a x \in U_{I} \cup U_{J}$. Now, let $x \in I \cup J$. Then $x \in I$ or $x \in J$. It results that $a x \in U_{I}$ or $a x \in U_{J}$ and so $a x \in U_{I} \cup U_{J}$. Hence $U_{I} \cup U_{J}=U_{I \cup J}$.

Therefore, by (i), (ii) and (iii), we obtain that $\Gamma$ is a topology on $E$.

Next, we present definition of linear submodules of an effect module and introduce another topology on $E$.

Definition 4.5. Let $I$ be an $E$-ideal of $E$. Then $I$ is called a linear $E$-ideal of $E$ if $I$ is also a total order set.

Example 4.6. In Example $4.2(i i), I$ and $J$ are linear $E$-ideals of $E$.

For every subset $I$ of $E$, we denote

$$
\begin{array}{r}
L_{I}=\{(x, y) \in E \times E: x+y \text { is defined and } \exists c \in I \text { that } x+c=y \\
\text { or } \exists d \in I \text { that } y+d=x\} .
\end{array}
$$

Let $L, K \subseteq E \times E$ such that $x+y$ be defined in them, for every $x, y$ in them. Then we denote

$$
L^{-1}=\{(y, x):(x, y) \in L\}, L(y)=\{x:(y, x) \in L, \text { for every } y \in E\}
$$

and

$$
L \circ K=\{(x, z): \exists y \in E \text { such that }(x, y) \in L \text { and }(y, z) \in K\} .
$$


Lemma 4.7. Let $I$ and $J$ be subsets of $E$.

(i) If $I \subseteq J$, then $L_{I} \subseteq L_{J}$.

If $E$ is an extended effect algebra and $I, J$ are $E$-ideals of $E$, then

(ii) $L_{I} \cap L_{J}=L_{I \cap J}$.

(iii) $L_{I} \cup L_{J} \subseteq L_{I} \circ L_{J}$.

If $E$ is an extended effect algebra and $I, J$ are linear $E$-ideals of $E$, then

(iv) $I \cap J$ is a linear E-ideal of $E$;

(v) $L_{I} \circ L_{I}=L_{I}$.

\section{PROOF:}

(i) The proof is clear.

(ii) Let $(x, y) \in L_{I} \cap L_{J}$. Then $(x, y) \in L_{I}$ and $(x, y) \in L_{J}$ and so $\left(x+c_{1}=y\right.$ or $\left.y+d_{1}=x\right)$ and $\left(x+c_{2}=y\right.$ or $\left.y+d_{2}=x\right)$, for $c_{1}, d_{1} \in I$ and $c_{2}, d_{2} \in J$. There are four possible cases:

Case (1): Let $x+c_{1}=y$ and $x+c_{2}=y$. Then $x+c_{1}=x+c_{2}$. Since "+" is cancellative, we have $c_{1}=c_{2} \in I \cap J$ and so $(x, y) \in L_{I \cap J}$.

Case (2): Let $x+c_{1}=y$ and $y+d_{2}=x$. Then $x \leq y$ and $y \leq x$ and so $x=y$. It means that $c_{1}=d_{2}=0 \in I \cap J$ and so $(x, y) \in L_{I \cap J}$.

Case (3): Let $y+d_{1}=x$ and $y+d_{2}=x$. The proof of this case is similar to the case (1).

Case (4): Let $y+d_{1}=x$ and $x+c_{2}=y$. The proof of this case is similar to the case (2). Hence $L_{I} \cap L_{J} \subseteq L_{I \cap J}$. It is easy to show that $L_{I \cap J} \subseteq L_{I} \cap L_{J}$. Therefore, $L_{I} \cap L_{J}=L_{I \cap J}$.

(iii) Let $(x, y) \in L_{I} \cup L_{J}$. Then $(x, y) \in L_{I}$ or $(x, y) \in L_{J}$. Let $(x, y) \in L_{I}$. Since $(y, y) \in L_{\{0\}} \subseteq L_{J}$, we have $(x, y) \in L_{I} \circ L_{J}$. Similarly, if $(x, y) \in L_{J}$, then $(x, y) \in L_{I} \circ L_{J}$. Thus, $L_{I} \cup L_{J} \subseteq L_{I} \circ L_{J}$.

(iv) The proof is clear.

(v) Let $(x, z) \in L_{I} \circ L_{I}$. Then there is $y \in E$ such that $(x, y) \in L_{I}$ and $(y, z) \in L_{I}$. Thus, $\left(x+c_{1}=y\right.$ or $\left.y+d_{1}=x\right)$ and $\left(x+c_{2}=z\right.$ or $z+d_{2}=y$ ), for $c_{1}, d_{1}, c_{2}, d_{2} \in I$. There are four possible cases: 
(1) Let $x+c_{1}=y$ and $y+c_{2}=z$. Then $x+c_{1}+c_{2}=z$. Since $c_{1}+c_{2} \in I$, we have $(x, z) \in L_{I}$.

(2) Let $x+c_{1}=y$ and $z+d_{2}=y$. Since $I$ is a linear set, we have $c_{1} \leq d_{2}$ or $d_{2} \leq c_{1}$. If $c_{1} \leq d_{2}$, then there is $e \in E$ such that $c_{1}+e=d_{2}$, thus $e \leq d_{2} \in I$, so $e \in I$. Also, we have $z+c_{1}+e=x+c_{1}$. So $z+e=x$ and so $(z, x) \in L_{I}$. Then $(x, z) \in\left(L_{I}\right)^{-1}=L_{I}$.

(3) Let $y+d_{1}=x$ and $y+c_{2}=z$. Then similar to (2), we can prove that $(x, z) \in\left(L_{I}\right)^{-1}=L_{I}$.

(4) $y+d_{1}=x$ and $z+d_{2}=y$. Similar to (1), we prove that $L_{I} \circ L_{I} \subseteq L_{I}$.

On the other hand, by (iii), it is clear that $L_{I} \subseteq L_{I} \circ L_{I}$. Therefore, $L_{I} \circ L_{I}=L_{I}$.

TheOREM 4.8. Let $E$ be an extended effect algebra, $\mathcal{I}$ be a family of all linear E-ideals of $E, K_{0}=\left\{L_{I}: I \in \mathcal{I}\right\}$ and

$$
\begin{gathered}
K=\{V \subseteq E \times E: x+y \text { is defined for every }(x, y) \in V \\
\text { and } \left.\exists L_{I} \in K_{0} \text { such that } L_{I} \subseteq V\right\} .
\end{gathered}
$$

Then

(1) If $V \in K$, then $V^{-1} \in K$.

(2) For every $V \in K$, there is $L \in K_{0}$ such that $L \circ L \subseteq V$.

(3) For every $V, L \in K$, we have $L \cap V \in K$.

(4) If $L \in K$ and $L \subseteq V \subseteq E \times E$ such that for any $(x, y) \in V, x+y$ is defined, then $V \in K$.

Proof: By Lemma 4.7, the result can obtain immediately.

Corollary 4.9. Consider the set $K$ in Theorem 4.8 and $T=\left\{L_{I}(x): I \in\right.$ $\mathcal{I}, x \in E\}$. Then

(i) $K$ is a base of a topology of $E \times E$.

(ii) $T$ is a base of a topology of $E$. 


\section{PROOF:}

(i) We should proof that (1) $E \times E=\bigcup_{V \in K} V$; (2) for any $V_{1}, V_{2} \in K$ and $x \in V_{1} \cap V_{2}$, there exists $V \in K$ such that $x \in V \subseteq V_{1} \cap V_{2}$.

(1) Let $(x, y) \in E \times E$. Then we can consider $V=L_{I}(x) \times L_{I}(y)=$ $\left\{(a, b):(x, a) \in L_{I}(x)\right.$ and $\left.(y, b) \in L_{I}(y)\right\}$. Since $(x, x) \in$ $L_{I}(x)$ and $(y, y) \in L_{I}(y)$, we have $(x, y) \in V$ and so $E \times E \subseteq$ $\bigcup_{V \in K} V$. Hence $E \times E=\bigcup_{V \in K} V$.

(2) Let $V_{1}, V_{2} \in K$ and $x \in V_{1} \cap V_{2}$. Then by Theorem 4.8 (3), we have $V_{1} \cap V_{2} \in K$ and so we consider $V=V_{1} \cap V_{2}$. Therefore, $K$ is a base of a topology of $E \times E$.

(ii) Similar to proof $(i)$, we should prove that $E=\bigcup_{x \in E} L_{I}(x)$ and there exists $V \in T$ with similar condition (2) in proof $(i)$. Let $x \in E$. Since $x=x+0$, we have $x \in L_{I}(x)$. Then $E \subseteq \bigcup_{x \in E} L_{I}(x)$ and so $E=\bigcup_{x \in E} L_{I}(x)$. Also, for $L_{I}(x), L_{I}(y) \in T$ and $x \in L_{I}(x) \cap L_{I}(y)$, by Lemma 4.7 (ii), we have $L_{I \cap J}=L_{I}(x) \cap L_{I}(y)$ and so we consider $V=L_{I}(x) \cap L_{I}(y)$. Therefore, $T$ is a base of a topology of $E$.

In following, we present definition of effect topological modules and we give a general example about them.

Definition 4.10. Let $E$ be a $P$-module. If $f: E \times E \longrightarrow E$ (defined by $f\left(e, e^{\prime}\right)=e+e^{\prime}$, for every $e, e^{\prime} \in E$, where $E \times E$ is multiplicative topology in $E$ ) and $\mu_{x}: E \longrightarrow E$ (defined by $\mu_{x}(e)=x e$, for every $e \in E$ and $x \in P$ ) are continuous under some topology $\tau$, then $(E, \tau)$ is called a topological effect module.

Example 4.11. Let $E$ be a $P$-module and $\left\{E_{n}: E_{n} \supseteq E_{n+1}, n \in \mathbf{N}\right\}$ be a decreasing sequence of proper submodules of $E$. Then it is routine to see that the collection

$$
\tau=\left\{V \subseteq E: \forall v \in V \exists n \in \mathbf{N} \text { such that } v+E_{n} \subseteq V\right\},
$$

where $V+E_{n}=\{v+e: v+e$ is defined in $E\}$ forms a topology on $E$. Also, $\mathcal{B}_{\tau}=\left\{x+E_{n}: x \in E, n \in \mathbf{N}\right\}$ forms a base for $\tau$. Now, we show that the addition "+" and the effect module multiplication are continuous under topology $\tau$. Consider $f: E \times E \longrightarrow E$ defined by $f\left(e, e^{\prime}\right)=e+e^{\prime}$, for $e, e^{\prime} \in E$ and a basic open set $e+E_{n} \in \mathcal{B}_{\tau}$. If $f^{-1}\left(e+E_{n}\right)=\emptyset$, then 
result holds trivially. If $f^{-1}\left(e+E_{n}\right) \neq \emptyset$, then it is easy to prove that $f^{-1}\left(e+E_{n}\right)$ is open and so $f$ is continuous. Finally, it is easy to show that the mapping $\mu_{x}: E \longrightarrow E$ defined by $\mu_{x}(e)=x e$ is continuous, for every $x \in P$. Therefore, $\tau$ force $E$ to be a topological effect module.

Next, we present definition of prime submodules in effect modules and we present two topology on them.

Definition 4.12. Let $E$ be a $P$-module and $I$ be a proper submodule of $E$. Then $I$ is called a prime submodule of $E$ if it satisfies in the following condition:

If $a x \in I$, then $a \in(I: E)$ or $x \in I$, for any $a \in P$ and $x \in E$.

The set of all prime submodules of $E$ is denoted by $\operatorname{Spec}_{P}(E)$.

Example 4.13. In Example 3.2 (iii), $I=\{0,1\}$ and $J=\{0,2\}$ are prime submodules of $E$ and $\{0\}$ is not a prime submodule of $E$. Note that $\operatorname{Spec}_{P}(E)=\{I, J\}$.

Definition 4.14. Let $E$ be a $P$-module and $\mathcal{T}(E)=\left\{\mathcal{V}(I): I \in S b_{P}(E)\right\}$, where $\mathcal{V}(I)=\left\{P \in \operatorname{Spec}_{P}(E): I \subseteq P\right\}$. If $\mathcal{T}(E)$ is closed under finite union, then $E$ is called a Top $P$-module.

Example 4.15.

(i) If $E$ is a $P$-module and $\operatorname{Spec}_{P}(E)=\emptyset$, then $E$ is a Top $P$-module.

(ii) By Example 4.13, $\operatorname{Spec}_{P}(E)=\{I, J\}$ and $\mathcal{T}(E)=\{\emptyset,\{I\},\{J\},\{I, J\}\}$. It is easy to see that $E$ is a Top $P$-module.

(iii) By Example 3.2 (iv), It is easy to see that $I=\{(0,0)\}, J=\{(0,0)$, $(0, x),(0,1)\}$ and $K=\{(0,0),(x, 0),(1,0)\}$ are prime submodules of $E$. We have $\mathcal{V}(I)=\{I\}, \mathcal{V}(J)=\{J\}$ and $\mathcal{V}(K)=\{K\}$. It is routine to see that $E$ is not a Top $P$-module.

Proposition 4.16. Let $E$ be a Top $P$-module. Then $\mathcal{T}(E)$ satisfies the axioms for closed sets in a topological space.

Proof: Clearly, $\mathcal{V}(E)=\emptyset$ and $\mathcal{V}(\{0\})=\operatorname{Spec}_{P}(E)$. It is enough to show that $\bigcap_{i \in I} \mathcal{V}\left(I_{i}\right)=\mathcal{V}\left(\bigvee_{i \in I} I_{i}\right)$, where $\bigvee_{i \in I} I_{i}=S u p\left\{I_{i}: i \in I\right\}$. Let $P \in \bigcap_{i \in I} \mathcal{V}\left(I_{i}\right)$. Then $P \in \mathcal{V}\left(I_{i}\right)$ and so $I_{i} \subseteq P$, for every $i \in I$. Hence $\bigvee_{i \in I} I_{i} \subseteq P$ and so $P \in \mathcal{V}\left(\bigvee_{i \in I} I_{i}\right)$. Thus $\bigcap_{i \in I} \mathcal{V}\left(I_{i}\right) \subseteq \mathcal{V}\left(\bigvee_{i \in I} I_{i}\right)$. On 
the other hand, we have $\mathcal{V}\left(\bigvee_{i \in I} I_{i}\right) \subseteq \bigcap_{i \in I} \mathcal{V}\left(I_{i}\right)$. Therefore, $\bigcap_{i \in I} \mathcal{V}\left(I_{i}\right)=$ $\mathcal{V}\left(\bigvee_{i \in I} I_{i}\right)$.

Remark 4.17. Let $E$ be a Top $P$-module. Then By Proposition $4.16, \mathcal{T}_{E}=$ $\left\{\mathcal{V}(I)^{c}: I \in S b_{P}(E)\right\}$ is a topology on $\operatorname{Spec}_{P}(E)$.

Definition 4.18. Let $E$ be a $P$-module and $K$ be a submodule of $E$. If $K$ is an intersection of some prime submodules of $E$, then $K$ is called a semiprime submodule of $E$.

Definition 4.19. Let $E$ and $F$ be two effect algebras. A mapping $f$ : $E \longrightarrow F$ is said to be a $P$-homomorphism if $(i) f$ is a homomorphism; (ii) $f(a x)=a f(x)$, for any $a \in P$ and $x \in E$. If $f$ is one to one (onto), then $f$ is called a $P$-monomorphism ( $P$-epimorphism) and if $f$ is onto and one to one, then $f$ is called a $P$-isomorphism.

LEMMA 4.20 .

(i) $E$ is a Top $P$-module if and only if for every prime submodule $K$ of $E, N \cap L \subseteq K$ implies that $N \subseteq K$ or $L \subseteq K(*)$, where $N, L$ are semiprime submodules of $E$.

(ii) Let $E$ and $F$ be two P-modules, $f: E \longrightarrow F$ be a P-isomorphism and $G$ be a prime submodule of $F$ satisfying $(*)$. Then $f^{-1}(G)$ is a prime submodule of $E$ satisfying $(*)$.

\section{PROOF:}

(i) Let $K$ be a prime submodule of $E, N$ and $L$ be semiprime submodules of $K$ such that $N \cap L \subseteq K$. Since $E$ is a Top $P$-module, there exists a submodule $J$ of $E$ such that $\mathcal{V}(N) \cup \mathcal{V}(L)=\mathcal{V}(J)$. Since $N$ is a semiprime submodule of $E, N=\bigcap_{i \in I} P_{i}$, where $\left\{P_{i}\right\}_{i \in I}$ is a family of prime submodules of $E$. Then $P_{i} \in \mathcal{V}(N)$, for any $i \in I$. Since $\mathcal{V}(N) \subseteq \mathcal{V}(J)$, we have $P_{i} \in \mathcal{V}(J)$. Hence $J \subseteq N$ and $J \subseteq L$ and so $J \subseteq N \cap L$. It follows that $\mathcal{V}(N \cap L) \subseteq \mathcal{V}(J)$. Now, we have $\mathcal{V}(N) \cup \mathcal{V}(L) \subseteq \mathcal{V}(N \cap L) \subseteq \mathcal{V}(J)=\mathcal{V}(N) \cup \mathcal{V}(L)$ and so $\mathcal{V}(N) \cup \mathcal{V}(L)=\mathcal{V}(N \cap L)$. It means that $K \in \mathcal{V}(N)$ or $K \in \mathcal{V}(L)$ and so $N \subseteq K$ or $L \subseteq K$. The proof of converse is routine.

(ii) The proof is routine. 
Theorem 4.21. Let $E$ and $F$ be two $P$-modules and $f: E \longrightarrow F$ be a $P$-isomorphism. If $\mathcal{T}_{F}$ is a topology on $\operatorname{Spec}_{P}(F)$, then $\mathcal{T}_{E}^{-1}=\left\{V(N)^{c}\right.$ : $\left.N \in \operatorname{Spec}_{P}(E)\right\}$ is a topology on $\operatorname{Spec}_{P}(E)$, where

$$
V(I)=\left\{f^{-1}(K): K \in \operatorname{Spec}_{P}(F) \text { and } f(I) \subseteq K\right\},
$$

for every $I \subseteq F$.

Proof: Since $\mathcal{T}_{F}$ is a topology on $\operatorname{Spec}_{P}(F), \mathcal{T}(F)$ is closed under finite union and so by Lemma $4.20(i), N \cap L \subseteq K$ implies that $N \subseteq K$ or $L \subseteq K$, for every prime submodule $K$ of $F$, where $N, L$ are semiprime submodules of $F$. We claim that $\mathcal{T}^{-1}(E)=\left\{V(N): N \in \operatorname{Spec}_{P}(E)\right\}$ is closed under finite unions. By Lemma $4.20(i i), f^{-1}(K)$ is a prime submodule of $E$, for every $K \in \operatorname{Spec}_{P}(F)$. The first, we prove that $f(G) \in \operatorname{Spec}_{P}(F)$, for every $G \in \operatorname{Spec}_{P}(E)$. Let $x, y \in f(G)$ and $x+y$ be defined in $F$. Clearly, there are $m, n \in G$ such that $x=f(m), y=f(n)$ and $f(m)+f(n)$ is defined in $F$. Since $f^{-1}$ is a $P$-homomorphism and $f(m)+f(n)$ is defined in $F$, we result that $f^{-1}(f(m))+f^{-1}(f(n))$ is defined in $\mathrm{E}$ and so $m+n$ is defined in $E$. It means that

$$
x+y=f(m)+f(n)=f(m+n) \in f(G) .
$$

Now, let $x \leq y$ and $y \in f(G)$, for any $x, y \in F$. Then there are $m \in G$ and $n \in E$ such that $x=f(m)$ and $y=f(n)$. Since $f(m) \leq f(n)$, there is $f(r)=c \in F$ such that $f(m)+f(r)=f(n)$, for $r \in E$ and so $f(m+r)=f(n)$. Hence $m+r=n$ and so $m \leq n \in G$. It means that $m \in G$ and so $x=f(m) \in G$. Thus, $f(G)$ is a submodule of $F$. It is routine to show that $f(G)$ is a prime submodule of $F$, for every $G \in \operatorname{Spec}_{P}(E)$. Then $f(N)=\bigcap_{G \in \operatorname{Spec}_{P}(E)} f(G)$ and $f(L)=\bigcap_{G^{\prime} \in \operatorname{Spec}_{P}(E)} f\left(G^{\prime}\right)$ are semiprime submodules of $F$. Hence by Lemma 4.20, $N \cap L \subseteq f^{-1}(G)$ implies that $N \subseteq f^{-1}(G)$ or $L \subseteq f^{-1}(G)$. Now, it is routine to see that $V(N) \cup V(L)=$ $V(N \cap L)$, for every semiprime submodules of $E$ and so by a straightforward way, we conclud that $\mathcal{T}^{-1}(E)$ is closed under finite unions. Therefore,

$$
\mathcal{T}_{E}^{-1}=\left\{V(N)^{c}: N \in \operatorname{Spec}_{P}(E)\right\}
$$

is a topology on $\operatorname{Spec}_{P}(E)$.

In following, we present topology on $\operatorname{Spec}_{P}(E)$ that is coarser than $\mathcal{T}_{E}$. Let $E$ be a $P$-module, $N$ be a submodule of $E$ and $J \subseteq P$. Then we denote: 


$$
\begin{aligned}
W(N)= & \left\{P \in \operatorname{Spec}_{P}(E):(N: E) \subseteq(P: E)\right\}, \\
\Upsilon_{E}= & \left\{W(N)^{c}: N \in S b_{P}(E)\right\} \\
J E= & \left\{x \in E: x \leq a_{1} x_{1}+\cdots+a_{n} x_{n}, \text { s.t. } \exists a_{1} \cdots, a_{n} \in J, x_{1}, \cdots, x_{n} \in E:\right. \\
& \left.a_{1} x_{1}+\cdots+a_{n} x_{n} \text { is defined in } E\right\}
\end{aligned}
$$

Lemma 4.22. Let $E$ be a $P$-module and $N$ be a submodule of $E$. Then $a . b \in(N: E)$, for every $a \in P$ and $b \in(N: E)$.

Proof: Let $a \in P$ and $b \in(N: E)$. Then $b E \subseteq N$ and so $b e \in N$, for every $e \in N$. Hence $(a . b) e=a(b e) \in N$ and so $a . b \in(N: E)$.

Theorem 4.23. Let E be a P-module. Then $\Upsilon_{E}$ is a topology on $\operatorname{Spec}_{P}(E)$.

Proof: It is clear that $W(N)=\emptyset$ and $W(\{0\})=\operatorname{Spec}_{P}(E)$. It is routine to see that $W(N) \cup W(M)=W(N \cap M)$, for every $N, M \in S b_{P}(E)$. We show that $\bigcap_{i \in I} W\left(N_{i}\right)=W(J E)$, where $J=\bigvee_{i \in I}\left(N_{i}: E\right)$. The first, we prove that $J E$ is a submodule of $E$. Let $a, b \in J E$ and $a+b$ is defined in E. Then

$$
a \leq a_{1} x_{1}+\cdots+a_{n} x_{n} \text { and } b \leq b_{1} y_{1}+\cdots+b_{m} y_{m}
$$

where $a_{1} x_{1}+\cdots+a_{n} x_{n}$ and $b_{1} y_{1}+\cdots+b_{m} y_{m}$ are defined in $E$, for some $a_{1}, \cdots, a_{n}, b_{1}, \cdots, b_{m} \in J$ and $x_{1}, \cdots, x_{n}, y_{1}, \cdots, y_{m} \in E$. So

$$
a+b \leq a_{1} x_{1}+\cdots+a_{n} x_{n}+b_{1} y_{1}+\cdots+b_{m} y_{m}
$$

If $a_{1} x_{1}+\cdots+a_{n} x_{n}+b_{1} y_{1}+\cdots+b_{m} y_{m}$ is not defined in $E$, then we can rewrite it by new $a_{i}$ 's and $b_{i}$ 's such that is defined in $E$ (since $a+b$ is defined in $E$, it is possible). Thus, $I_{1}$ is true. Note that $\left(I_{2}\right)$ is clear. Now, let $e \in P$ and $a \in J E$. Then $a \leq a_{1} x_{1}+\cdots+a_{n} x_{n}$, where $a_{1} x_{1}+\cdots+a_{n} x_{n}$ is defined, for any $a_{1}, \cdots, a_{n} \in J$ and $x_{1}, \cdots, x_{n} \in E$. Since $a \leq a_{1} x_{1}+\cdots+a_{n} x_{n}$, there is $c \in E$ such that $a+c=a_{1} x_{1}+\cdots+a_{n} x_{n}$ and so by (PE2) and (PE3),

$$
e a+e c=\left(e . a_{1}\right) x_{1}+\cdots+\left(e . a_{n}\right) x_{n}
$$

It means that $e a \leq\left(e . a_{1}\right) x_{1}+\cdots+\left(e . a_{n}\right) x_{n}$, where by Lemma 4.22, $\left(e . a_{1}\right), \cdots,\left(e . a_{n}\right) \in J$ and so $e a \in J E$. Hence $J E$ is a submodule of $E$. Now, it is routine to see that $\bigcap_{i \in I} W\left(N_{i}\right)=W(J E)$. Therefore, $\Upsilon_{E}$ satisfies the axioms of topology defined by open sets. 
Example 4.24.

(i) In Example 3.2 (iii), we have $\operatorname{Spec}_{P}(E)=\{I, J\}$. Then $W(I)=\{I\}$, $W(J)=\{J\}, W(\emptyset)=\operatorname{Spec}_{P}(E)$ and $W(E)=\{\emptyset\}$. Then $\Upsilon_{E}=$ $\{\emptyset,\{I\},\{J\},\{I, J\}\}$ is topology on $\operatorname{Spec}_{P}(E)$.

(ii) In Example 4.15 (iii), we have $\operatorname{Spec}_{P}(E)=\{I, J, K\}$. Then $W(E)=\emptyset$,

$$
W(I)=W(J)=W(K)=\operatorname{Spec}_{P}(E), \Upsilon_{E}=\left\{\emptyset, \operatorname{Spec}_{P}(E)\right\} .
$$

Therefore, $\Upsilon_{E}$ is topology on $\operatorname{Spec}_{P}(E)$.

\section{Conclusion}

Effect algebras generalize many examples of quantum structures, like Boolean algebras, orthomodular lattices or posets, orthoalgebras, $M V$ algebras, etc. Recently, module structures have been defined over some algebraic structures, and some researches have been interested in this topic. We presented definition of effect modules. Next researchers can study free effect modules, projective (injective) modules and many of the other concepts of modules. In the study of effect algebras (or more general, quantum structures) as carriers of states and probability measures, an important tool is the study of topologies on them. Also, the studying of certain topological properties of algebraic structures characterize also their certain algebraic properties. We studied and introduced some topologies on effect modules. We wish that the obtained results can encourage us to continue this long way. In fact, we hope that we could open new fields to anyone that is interested to studying and development of modules.

\section{References}

[1] M. K. Bennett, D. J. Foulis, Phi-symmetric effect algebras, Foundations of Physics, vol. 25 (1995), pp. 1699-1722, DOI: http://dx.doi.org/10.1007/ BF02057883.

[2] R. A. Borzooei, A. Dvurečenskij, A. H. Sharafi, Material Implications in Lattice Effect Algebras, Information Sciences, vol. 433-434 (2018), pp. 233-240, DOI: http://dx.doi.org/10.1016/j.ins.2017.12.049. 
[3] R. A. Borzooei, S. S. Goraghani, Free Extended BCK-Module, Iranian Journal of Mathematical Science and Informatics, vol. 10(2) (2015), pp. 29-43, DOI: http://dx.doi.org/10.7508/ijmsi.2015.02.004.

[4] R. A. Borzooei, S. S. Goraghani, Free MV-modules, Journal of Intelligent and Fuzzy System, vol. 31(1) (2016), pp. 151-161, DOI: http://dx.doi. org/10.3233/IFS-162128.

[5] R. A. Borzooei, N. Kohestani, G. R. Rezaei, Metrizability on (Semi)topological BL-algebra, Soft Computing, vol. 16(10) (2012), pp. 1681-1690, DOI: http://dx.doi.org/10.1007/s00500-012-0852-2.

[6] R. A. Borzooei, G. R. Rezaei, N. Kohestani, On (semi)topological BLalgebra, Iranian Journal of Mathematical Science and Informatics, vol. 6(1) (2011), pp. 59-77, DOI: http://dx.doi.org/10.7508/ijmsi.2011.01. 006.

[7] R. A. Borzooei, O. Zahiri, Topology on BL-algebras, Fuzzy Sets and Systems, vol. 289 (2016), pp. 137-150, DOI: http://dx.doi.org/10.1016/j.fss. 2014.11.014.

[8] R. Cignoli, I. M. L. D'Ottaviano, D. Mundici, Algebraic Foundations of Many-valued Reasoning, Kluwer Academic-Dordrecht (2000).

[9] A. Di Nola, P. Flondor, I. Leustean, $M V$-modules, Journal of Algebra, vol. 267 (2003), pp. 21-40, DOI: http://dx.doi.org/10.1016/S0021-8693(03) 00332-6.

[10] A. Di Nola, C. Russo, Semiring and semimodules issues in $M V$-algebras, Communications in Algebra, vol. 41(3) (2013), pp. 1017-1048, DOI: http://dx.doi.org/10.1080/00927872.2011.610074.

[11] A. Dvurečenskij, New trends in quantum structures, Kluwer Academic/Ister Science-Dordrecht/Bratislava (2000).

[12] A. Dvurečenskij, Product effect algebras, International Journal of Theoretical Physics, vol. 41(10) (2002), pp. 193-215, DOI: http://dx.doi.org/ 10.1023/A:1021017905403.

[13] A. Dvurečenskij, States on Pseudo effect algebras and integrals, Foundations of Physics, vol. 41 (2011), pp. 1143-1162, DOI: http://dx.doi.org/ 10.1007/s10701-011-9537-4.

[14] A. Dvurečenskij, T. Vetterlein, Pseudo effect algebras. II. Group represetation, International Journal of Theoretical Physics, vol. 40 (2001), pp. 703-726, DOI: http://dx.doi.org/10.1023/A:1004144832348. 
[15] F. Forouzesh, E. Eslami, A. B. Saeid, Spectral topology on $M V$-modules, New Mathematics and Natural Computation, vol. 11(1) (2015), pp. 13-33, DOI: http://dx.doi.org/10.1142/S1793005715500027.

[16] D. J. Foulis, M. K. Bennett, Effect algebras and unsharp quantum logics, Foundations of Physics, vol. 24(10) (1994), pp. 1331-1352, DOI: http: //dx.doi.org/10.1007/BF02283036.

[17] S. S. Goraghani, R. A. Borzooei, Results on Prime Ideals in PMV-algebras and $M V$-modules, Italian Journal of Pure and Applied Mathematics, vol. 37 (2017), pp. 183-196.

[18] M. R. Rakhshani, R. A. Borzooei, G. R. Rezaei, On topological effect algebras, Italian Journal of Pure and Applied Mathematics, vol. 39 (2018), pp. 312-325.

\section{Simin Saidi Goraghani}

Farhangian University

Department of Mathematics

Tehran, Iran

e-mail: siminsaidi@yahoo.com

\section{Rajab Ali Borzooei}

Shahid Beheshti University

Department of Mathematics

Tehran, Iran

e-mail: borzooei@sbu.ac.ir 